\title{
UAV Image Fast Splicing Correction Based on Secondary Information
}

\author{
Fanlong Kong and Jingjia Gu*
}

East China Normal University, Shanghai, 200062, P.R. China

\begin{abstract}
For UAV system only using the secondary data without the control of node data, the automatic image splicing and quickly correcting method is proposed based on the UAV image blocking of pseudo center point secondary data to realize the fast image splicing and correcting handling. SIFT algorithm is adopted for high accurate and high efficient automatic splicing; the pseudo center point of the secondary data is analyzed to correct the blocking images. Experimental results show that secondary data with pseudo center point combining with SIFT algorithm for processing not only avoids the occurrence of image visual identity but has a high correcting accuracy.
\end{abstract}

Keywords: Fast splicing, image correction, sift algorithm, UAV.

\section{INTRODUCTION}

As an important means to obtain spatial data, UAV can collect the information in dangerous environment in a riskfree manner, which is featured by real-time, flexibility, high resolution and low cost $[1,2]$. Therefore, it is widely used in emergency monitoring and post-disaster reconstruction, in particular used in on-site emergency response [3]. UAV has the ability of fast data collection and data transmission. Currently, there is no relatively mature image processing system for UAV in China [4], and corresponding image processing software is also rare. UAV which is based on traditional photogrammetry has relatively high precision of image processing technology, however, it has very rigorous requirement on original data [5]. Therefore, highly precise attitude parameters and data of ground control point must be acquired. The flying quality of UAV is significantly affected by lowaltitude flight, and traditional aerial triangulation cannot meet its requirement. So relevant data of UAV's graphic processing cannot be obtained by means of traditional aerial photography. Therefore, it is quite urgent to research a UAV image processing algorithm without data of ground control node. This article proposes SIFT algorithm applied in image splicing, which is featured by unchanged local features, high precision and low efficiency $[6,7]$. To solve the problems of low efficiency of SIFT algorithm, it adopts an automatic image slicing method by secondary data.

\section{DATA ACQUISITION}

The test site of the UAV is located in a basin, where the climate is torrid and wet with frequent clouds and vapor; therefore, it can only fly in sunny days with low wind speed. The side of UAV along the river valley is stable when it flies at the average altitude of $660 \mathrm{~m}$. The total flying area is 35 $\mathrm{m}^{2}$. The key parameters of UAV platform are shown in Tables $\mathbf{1}$ and $\mathbf{2}$.
Table 1. The key parameters of UAV platform.

\begin{tabular}{|c|c|}
\hline Item & Parameter \\
\hline \hline Length $/ m$ & 1.2 \\
\hline Flight height $/ m$ & $400-1000$ \\
\hline Weight $/ \mathrm{kg}$ & 4.5 \\
\hline Speed $/(\mathrm{km} / \mathrm{h})$ & $70-110$ \\
\hline Flight time $/ \mathrm{min}$ & 60 \\
\hline Control mode & Automatic control \\
\hline Navigating mode & GPS \\
\hline Sensor & Digital camera \\
\hline
\end{tabular}

Table 2. Key parameters of the UAV sensor.

\begin{tabular}{|c|c|}
\hline Item & Parameter \\
\hline \hline Image sensor & Ricoh digital type \\
\hline Resolution & 10000000 \\
\hline Focus lens $/ \mathrm{mm}$ & 28 \\
\hline CCD size $/ \mathrm{m}$ & $(1 / 1.75) \times 3048$ \\
\hline Document size/ pixel & $3680 \times 2376$ \\
\hline Record format & JPEG \\
\hline
\end{tabular}

The transverse image overlapping rate and longitudinal image overlapping rate of photos that UAV takes at $880 \mathrm{~m}$ are $30 \%$ and $80 \%$ respectively. Of 3456 photos (the size of each photo is 300 thousand bytes) taken by two UAVs, each has a group of secondary data. The secondary data recorded by UAVs includes image sequence number, longitude and latitude coordinates, speed, flight height and orientation angle recorded by GPS. 


\section{DATA ANALYSIS}

\subsection{Image Data}

(1) Since it uses common digital cameras, the images have very small frames and quite large sizes. To increase the resolution of the images, the flight height must be decreased, which will result in small coverage area of the images.

The formula of the resolution of images can be obtained from serial photography standard 1:500, 1:1000 and 1:2000. According to the relationship among the focal distance, flight height and resolution, the formula is as follow:

$\frac{f}{H}=\frac{C}{A}$

In the above-mentioned formula $f$ : focal distance, $H$ : flight height, $C$ : CCD size; $A$ : ground coverage area; $A$ can be worked out by the formula $A=$ pixel number $\times$ resolution.

The parameters of the UAV sensor is shown in Table 2 , with the parameter of focal distance determined during shooting. Focal distance $5.9 \mathrm{~mm}$, CCD size $1 / 1.75$, image size $3648 \times 2376$. The flight height measured by GPS is $220 \mathrm{~m}$, substitute the said data into formula (1), resolution $R=0.08$ $m$. The coverage area of an image is $0.005 \mathrm{~m}^{2}$.

It will cost a lot of time to process 3000 images one by one by normal processing means.

(2) The shooting route is an irregular curve. Import the extracted quantity and coordinate data into Excel sheet, then open it via ArcGIS software, and it will finally outputs a Shape file document of flight path [8]. The curve in the picture represents the flight path, and the number represents the image shot.

It can be seen from Fig. (1) that the path of UAV is irregular, which causes the overlap of the images, in particularly the overlapping rate on the two transverse images which are more different.

(3) Large images are prone to be deformed. Since common digital cameras are used, and the flight attitude is unstable, the highlighting relationship between a single image and the ground space is complicated, and the geometrical relationship between images is unstable, so Len of image distorts seriously. The different comparison levels between light and shadow of images lead to large transformation of image inclination. Meanwhile, since it is in low altitude flight, the fluctuant ground will affect the resolution of the images.

\subsection{Secondary Data}

The recorded secondary information of the system record shall be exported and synthesized to the flight route map as shown in Fig. (1).

In view of the load limit and costs of UAV, the precision of the GPS installed on UAV is only $10 \mathrm{~m}$, and the auxiliary coordinate point recorded by GPS is not in the center of the image, the orientation angle is only accurate to degree.

There is no other data for this experiment except the coordinate data on the images and Google Map. It's difficult to obtain new data after the earthquake, and the resolution of Google map is rather low, we can only identify the rivers and buildings on that.

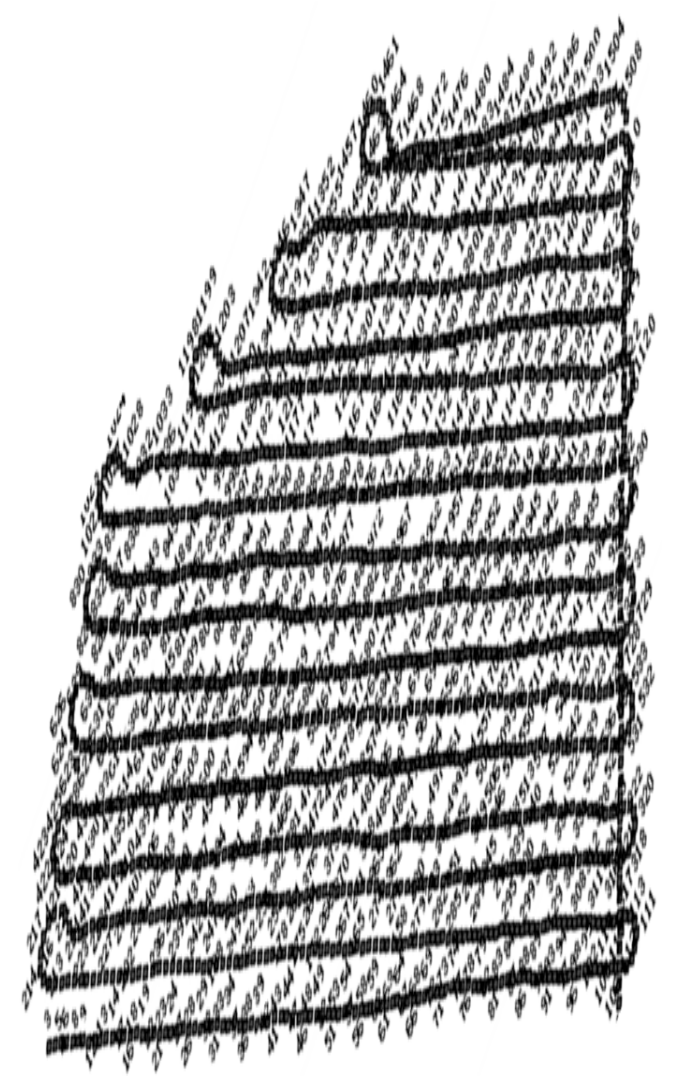

Fig. (1). Flight route map.

\section{IMAGE PROCESSING}

\subsection{Feature Extraction}

The image features are obtained by size conversion. However, for UAV, there is a lot of data need to process for original image processing; UAV is not capable to provide amplification processing for the images. In the experiment, we get approximate 8000 feature points after amplifying an image of $912 \times 648$, which is unimaginable for UAV. So we will process original images in the following experiments.

When using SIFT feature matching algorithm, the number of layers adopted in the process of feature point extracting shall meet differential Gauss Pyramid after adding two layers, and shall meet Gauss-shaped Pyramid after adding three layers. SIFT feature matching is as shown in Fig. (2).

This article sets the three images of wild land, urban buildings and highways as the study objects for the experiment. We analyze the image feature matching effect of UAV by using the images with the size of $912 \times 648$. The image feature matching effect of roads' images of several cameras is as shown in Table 3. The matching point pairs removed of gross error are defined as matching point pairs. The matching success rate shall be the ratio of the number of successful matching point pairs to the number of successful matching point pairs of either image (which is less).

As is known through analysis on Table $\mathbf{3}$, the number and time of feature extracting points are significantly related to the layers we set before. When we set more layers, we will get more feature points, and of course, we will need more time accordingly, however, the success rate of matching is 


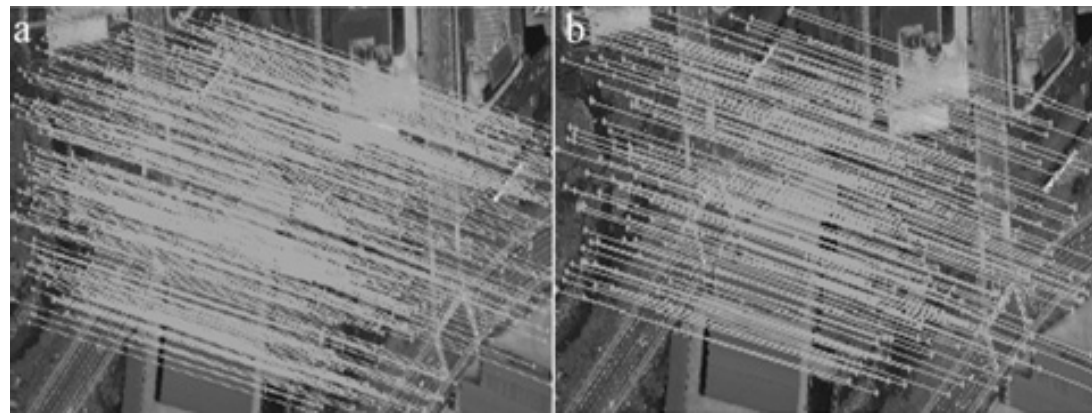

Fig. (2). SIFT feature matching results. (a) Three layers six orders; (b) One layer one order.

Table 3. Influence of different sizes on the image matching of UAV.

\begin{tabular}{|c|c|c|c|c|c|c|c|c|}
\hline \multirow{2}{*}{ Size Setting } & \multicolumn{2}{|c|}{ No. of Feature Points } & \multicolumn{2}{c|}{ Time for Proposing Features /s } & \multicolumn{3}{c|}{ Matching Point Pairs } \\
\cline { 2 - 8 } & Image 1 & Image 2 & Image 1 & Image 2 & $\begin{array}{c}\text { Time- } \\
\text { Consuming/s }\end{array}$ & $\begin{array}{c}\text { With Gross } \\
\text { Error }\end{array}$ & $\begin{array}{c}\text { Without } \\
\text { Gross Error }\end{array}$ & $\begin{array}{c}\text { Accuracy } \\
\text { Rate/(\%) }\end{array}$ \\
\hline \hline $\begin{array}{c}3 \text { layers 6 } \\
\text { orders }\end{array}$ & 1912 & 2279 & 6.202 & 6.436 & 4.172 & 1059 & 832 \\
\hline $\begin{array}{c}1 \text { layers 6 } \\
\text { orders }\end{array}$ & 372 & 471 & 2.596 & 2.828 & 0.203 & 220 & 194 \\
\hline $\begin{array}{c}3 \text { layers 3 } \\
\text { orders }\end{array}$ & 1885 & 2249 & 5.934 & 6.396 & 3.765 & 1047 & 829 \\
\hline $\begin{array}{c}1 \text { layer 3 } \\
\text { orders }\end{array}$ & 366 & 466 & 2.552 & 2.751 & 0.157 & 217 & 198 \\
\hline $\begin{array}{c}1 \text { layer 1 } \\
\text { order }\end{array}$ & 245 & 324 & 1.813 & 2.094 & 0.078 & 147 & 54.1 \\
\hline
\end{tabular}

not so high. Its reason is that much time is wasted on the points with no practical meaning during matching process. When we set the order $=1$, layer $=1$, we can successfully match 131 pairs of matching points.

Table 3 lists the original matching point pairs and the matching point pairs after removing gross error by means of RANSAC algorithm in details. In this article, the images of roads are set as the study objects, and we set 1.5 pixels as the distance threshold value of RANSAC algorithm.

The images need to correct since they have anamorphous. Generally, there are two methods to correct the images: geometric correction is to correct anamorphous due to the said systemic and non-systematic factors, thus realizing complete registration and matching with standard image or the map with specific projection and coordinated system, and enabling it to have the features of spatial parameters. Orthorectification is one of geometric corrections which are mainly used for processing aerial photos [9]. Only using geometric correction is coarser, and Ortho-rectification is more precise in processing aerial photos.

\subsection{Image Automatic Splicing and Correction}

Currently, there are two image splicing methods: the first one is based on coordination and uses flight attitude parameters and control point data. The other method is based on image feature matching and uses relevant indexes for wavelet analysis, SITT and Fourier transform etc.
Since only some secondary data is recorded (Shown in Table 4), and there are abundant data needs to process, the small images can only be processed by means of image matching algorithm. Since there is $80 \%$ longitudinal overlapping ratio, the overlapping of image can only be corrected after being spliced based on secondary data. In order to increase the speed and obtain the whole aerial images, two images shall be selected at the same direction, which can better ensure the overlapping rate (maximum overlapping rate is $80 \% \times 80 \% \times 80 \%$ ), and increase the image processing speed simultaneously.

Firstly, the optimal rows and lines of image automatic splicing in small areas should be determined by experiments. The determination standards include splicing precision and efficiency.

As tested in many experiments, $15 \times 5$ (relative longitudinal: 15; lateral 5) is most efficient. Meanwhile, to increase the splicing results among granules, the overlapping rate among granules shall be controlled within $35 \%$. The patches are spliced automatically, whose results can be increased by $5 \%$ by tuning the image size and overlapping rate. The complete processing procedure is shown in Fig. (3).

The image processing procedure is conducted by means of SIFT algorithm which can extract the local features of an images and stays unchanged when rotating, zooming and changing luminosity, and keeps stable affine transformation and noise when changing viewing angle [10-12]. It can make fast image slicing and accuractly extract data in characteristic 
Table 4. Secondary data recorded by the UAV system.

\begin{tabular}{|c|c|c|c|c|}
\hline $\mathbf{S} / \mathbf{N}$ & Latitude & Longitude & Speed & Height \\
\hline 0001 & $31^{\circ} 27.235^{\prime}$ & $104^{\circ} 10.535^{\prime}$ & V000 & H0682 \\
\hline 0002 & $31^{\circ} 27.235^{\prime}$ & $104^{\circ} 10.534^{\prime}$ & V000 & H0682 \\
\hline 0003 & $31^{\circ} 26.984^{\prime}$ & $104^{\circ} 10.183^{\prime}$ & V044 & H0874 \\
\hline 0004 & $31^{\circ} 26.965^{\prime}$ & $104^{\circ} 10.202^{\prime}$ & V043 & H0872 \\
\hline 0005 & $31^{\circ} 26.946^{\prime}$ & $104^{\circ} 10.220^{\prime}$ & V041 & H0871 \\
\hline i & $\begin{array}{l}1 \\
1\end{array}$ & $\begin{array}{l}1 \\
1\end{array}$ & $\begin{array}{l}1 \\
1\end{array}$ & $\begin{array}{l}1 \\
1\end{array}$ \\
\hline 3455 & $31^{\circ} 27.031^{\prime}$ & $104^{\circ} 10.496^{\prime}$ & V051 & H0887 \\
\hline 3456 & $31^{\circ} 27.002^{\prime}$ & $104^{\circ} 10.497^{\prime}$ & V050 & H0888 \\
\hline
\end{tabular}

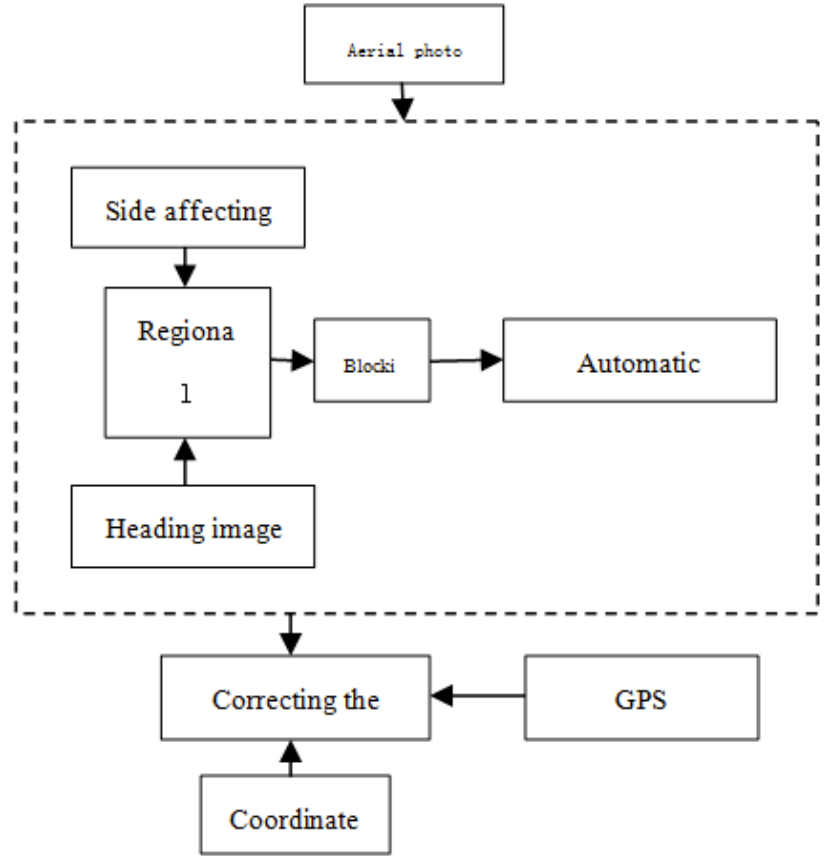

Fig. (3). Image processing flow chart.

database, therefore, it is very suitable for image process of expensive and easily deformed UAV.

The feature matching process of SIFT algorithm:

$$
\begin{aligned}
& G(x, y, \sigma)=\frac{1}{2 \pi \sigma^{2}} \exp \left[-\left(x^{2}+y^{2}\right) /\left(2 \sigma^{2}\right)\right] \\
& L(x, y, \sigma)=G(x, y, \sigma) * I(x, y)
\end{aligned}
$$

Different dimension spaces are built. In the formula, $x, y$ represent the coordinates of the points, $\sigma$ represents dimensional space, $G(x, y, \sigma)$ represents Gaussian function, $L(x, y, \sigma)$ represents dimensional space, * represents convolution operation. Different dimension spaces are built by changing the value of $\sigma$.

In Fig. (4), $s$ represents the sample of different dimension spaces. The coordinate points can be clearly position by DOG arithmetic after the establishment of scale space. When the feature coordinate point is determined, it shall be determined whether it is the feature coordinate point by comparing the coordinate point with 8 points surrounding it and the similar coordinate points in near spaces. The parameters of each coordinate point can be obtained by features of the adjacent pixelsvergence direction, which can keep the features of invariant rotation.

When establishing SIFT feature matching, the next thing is to determine the similarities of these features.

Finding the first coordinate point in the first image, and finding the second coordinate point in the second image, Euclidean distance between them is the nearest. The rate of the second one dividing the minimum distance is smaller than that of the first one and then the coordinate point will successfully match the nearest point. In Fig. (5), the end points of the two lines will be the matching coordinate points.

RANSAC algorithm is adopted to delete some wrong coordinate points, with at least four pairs of coordinate points retained, so as to establish bilinear transformation matrix:

$$
\begin{aligned}
& X=a_{1} x+b_{1} y+c_{1} x y+d_{1} \\
& Y=a_{2} x+b_{2} y+c_{2} x y+d_{2}
\end{aligned}
$$

Adjacent images can be spliced in line with the parameters of transformation matrix. The transformation matrix of the two images as follows:

$$
M=\left[\begin{array}{cccc}
0.999168 & -0.0386002 & 0.000131057 & 572.8177 \\
0.0386011 & 0.999255 & -0.000200111 & -34.324655
\end{array}\right]
$$

As seen from the matrix, zooming distortion factor is very small, however, there is certain rotation distortion.

\section{EXPERIMENT AND ANALYSIS}

The images shall be corrected by using the secondary data recorded by GPS after splicing. The position of the coordinate points recorded by GPS is not located in the image center, however, the relative position of GPS and the camera is invariant in the same direction, the coordinate points recorded by GPS can be deemed as the false central point. 


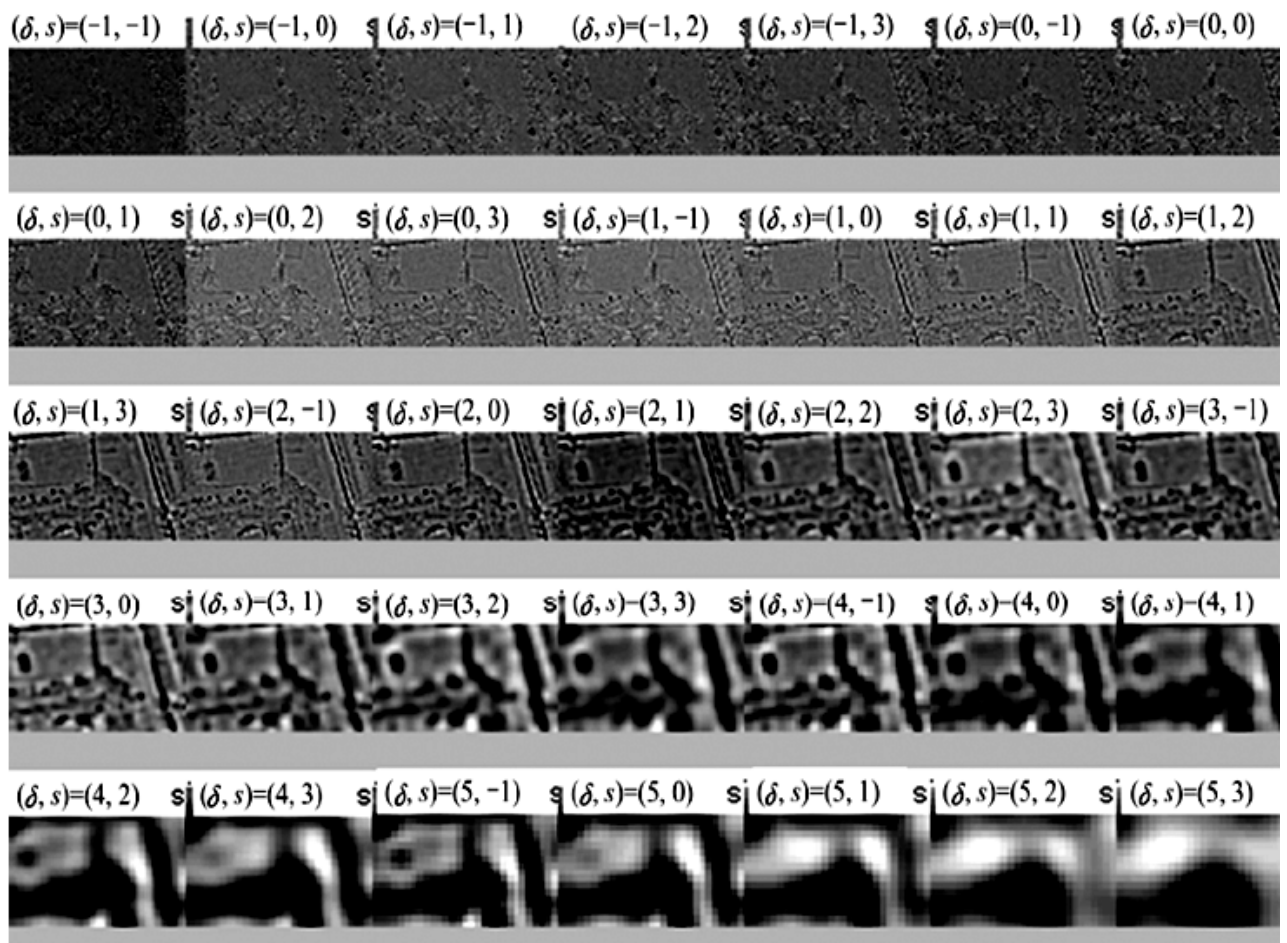

Fig. (4). Image features under different dimension spaces.

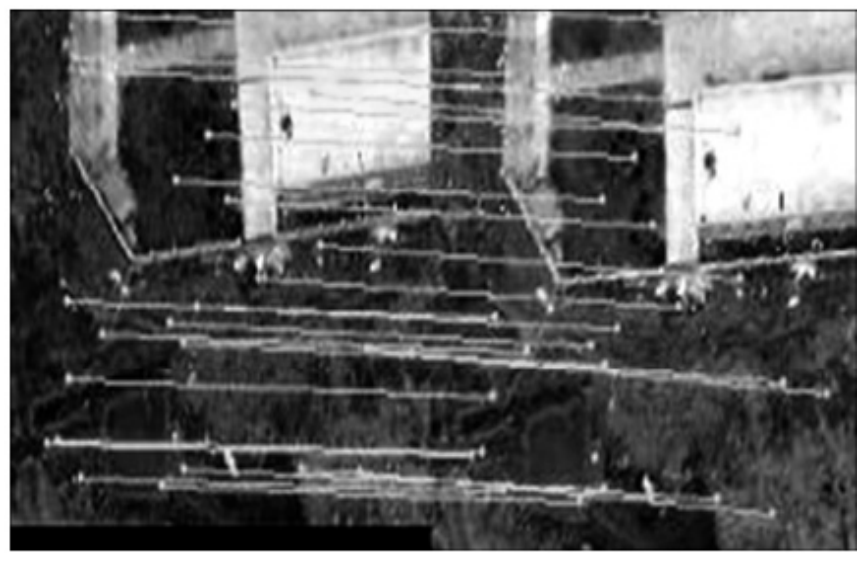

Fig. (5). Matching results of the feature coordinate point.

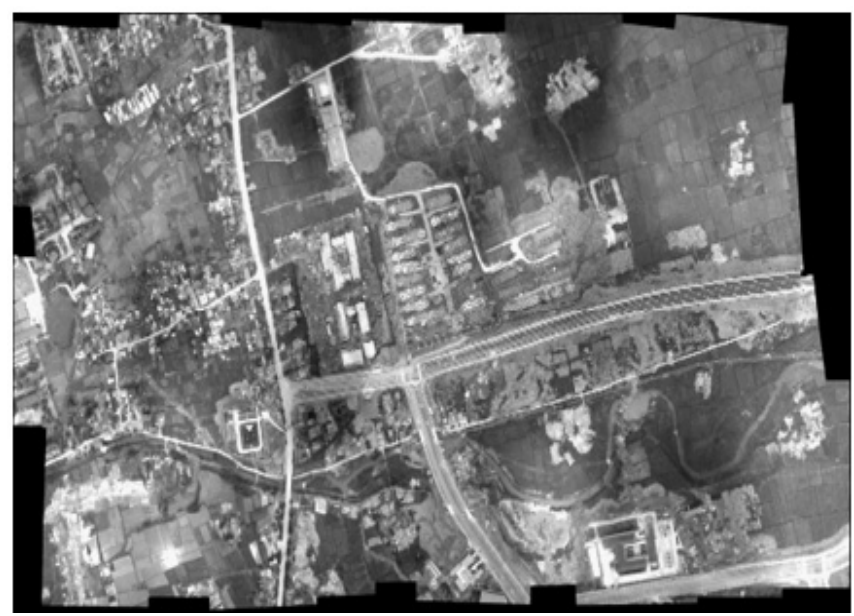

Fig. (6). Image before correction.

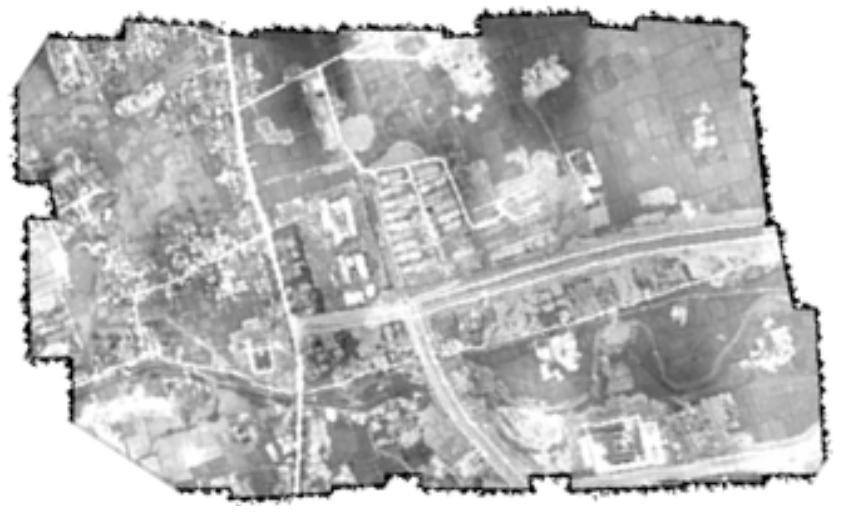

Fig. (7). Image after correction.

In this experiment, the coordinate point in the flight direction of top left is used for correcting the image. The image before correcting is shown in Fig. (6), and the image after correcting is shown in Fig. (7). From Fig. (7), we can see that the main factors affecting image splicing such as roads and river, etc. are perfect without visual mismatching. Since the corrected images are provided with coordinate information, it will be easier to splice them. Splicing these correct blocks into a large map will provide effective data source for the personnel's sorting and correction.

\section{CONCLUSION}

The goal of researching a method to fast process the images only by the secondary data of the false central point recorded by UAV is to realize fast image automatic splicing and image correction in large area.

Firstly, the data based on flight route can be divided into many blocks, then SIFT algorithm will be adopted to con- 
duct highly accurate and highly efficient automatic splicing. Finally, the false central point of the blocked images can be corrected by analyzing the secondary data.

The splicing and correction of all data in the experiment can be completed within 2 seconds, and a completed spliced image in the experimental area shall have high precision without visual mismatching.

The quantitative analysis for the splicing accuracy of the images haven't completed due to data limit, which is very important to the application of results.

\section{CONFLICT OF INTEREST}

The authors confirm that this article content has no conflict of interest.

\section{ACKNOWLEDGEMENTS}

Declared none.

\section{REFERENCES}

[1] B. Wang, Z. Gong, and K. Jin, "Improved UAV image mosaic method," Computer Engineering and Applications, vol. 47, 184187,2011
[2] J. He, Y. Li, H. Lu, and Z. Ren, "Research of UVA image quality evaluation and geometry processing," Bulletin of Surveying and Mapping, vol. 4, pp. 22-24, 2010.

[3] K. Mikolajczyk, and C. Schmid, "A performance evaluation of local descriptors," IEEE Transactions on Pattern Analysis and Machine Intelligence, vol. 27, pp. 1615-1630, 2012.

[4] F. Imado, S. Abe, and T. Kinoshita, "The development of threedimensional scale model UAV Simulator," SICE-ICASE International Joint Conference, vol. 4921-4925, 2006.

[5] H. Bay, A. Ess, T. Tuytelaars, and L. V. Gool, "Speeded-up robust features (SURF)," Computer Vision and Image Understanding, vol 110, pp. 346-359, 2008.

[6] C. Li, and L. Ma, "A new framework for feature descriptor based on SIFT," Pattern Recognition Letters, vol. 30, pp. 544-557, 2009.

[7] X. Yuan, G. Peng, and L. Wang, "GPU-based real time image registration with variant SIFT," Computer Science, vol. 38, pp. 300-303, 2011.

[8] J. He, and Y. Li, "UAV image mosaic based on optimal path," Science of Surveying and Mapping, vol. 36, pp. 152-154, 2011.

[9] Y. Di, Y. Chen, Y. Chen, and Y. Chen, "Image mosaic algorithm of unmanned aerial vehicle," Application Research of Computers, vol. 31, pp. 170-174, 2011.

[10] B. Wang, and W. Wang, "Fitting image mosaic algorithm of UAV based on local correspondence," Journal of China University of Petroleum, vol. 33, pp. 169-172, 2009.

[11] Y. Cheng, D. Xue, and X. Han, "Fast image mosaic based on wavelet transform for remote sensing," Journal of Northeastern University, vol. 29, pp. 1385-1388, 2008 .

[12] Y. Shen, J. Liu, L. Wu, F. Li, and Z. Wang, "Reconstruction of disaster scene from UAV images and flight-control data," Geography and Geo-Information Science, vol. 27, pp. 13-17, 2011.

Received: September 22, 2014

Revised: November 30, 2014

Accepted: December 02, 2014

(C) Kong and $\mathrm{Gu}$; Licensee Bentham Open.

This is an open access article licensed under the terms of the Creative Commons Attribution Non-Commercial License (http://creativecommons.org/licenses/by-nc/3.0/) which permits unrestricted, non-commercial use, distribution and reproduction in any medium, provided the work is properly cited. 